\title{
Cross-Modal Plasticity in Higher-Order Auditory Cortex of Congenitally Deaf Cats Does Not Limit Auditory Responsiveness to Cochlear Implants
}

\author{
Rüdiger Land, ${ }^{1}$ Peter Baumhoff, ${ }^{1}$ Jochen Tillein, ${ }^{1,2}$ Stephen G. Lomber, ${ }^{3}$ Peter Hubka, ${ }^{1}$ and Andrej Kral ${ }^{1,4}$ \\ ${ }^{1}$ Institute of AudioNeuroTechnology and Department of Experimental Otology, Clinics of Otolaryngology, Hannover Medical School, Hannover 30625, \\ Germany, ${ }^{2}$ Clinics of Otolaryngology, School of Medicine, J.W. Goethe University 60590, Frankfurt am Main, Germany, ${ }^{3}$ Brain and Mind Institute and \\ Department of Physiology and Pharmacology, University of Western Ontario, London, Ontario N6A 5C2, Canada, and ${ }^{4}$ School of Behavioral and Brain \\ Sciences, The University of Texas at Dallas, Dallas, Texas 75080
}

Congenital sensory deprivation can lead to reorganization of the deprived cortical regions by another sensory system. Such cross-modal reorganization may either compete with or complement the "original" inputs to the deprived area after sensory restoration and can thus be either adverse or beneficial for sensory restoration. In congenital deafness, a previous inactivation study documented that supranormal visual behavior was mediated by higher-order auditory fields in congenitally deaf cats (CDCs). However, both the auditory responsiveness of "deaf" higherorder fields and interactions between the reorganized and the original sensory input remain unknown. Here, we studied a higher-order auditory field responsible for the supranormal visual function in CDCs, the auditory dorsal zone (DZ). Hearing cats and visual cortical areas served as a control. Using mapping with microelectrode arrays, we demonstrate spatially scattered visual (cross-modal) responsiveness in the DZ, but show that this did not interfere substantially with robust auditory responsiveness elicited through cochlear implants. Visually responsive and auditory-responsive neurons in the deaf auditory cortex formed two distinct populations that did not show bimodal interactions. Therefore, cross-modal plasticity in the deaf higher-order auditory cortex had limited effects on auditory inputs. The moderate number of scattered cross-modally responsive neurons could be the consequence of exuberant connections formed during development that were not pruned postnatally in deaf cats. Although juvenile brain circuits are modified extensively by experience, the main driving input to the cross-modally (visually) reorganized higher-order auditory cortex remained auditory in congenital deafness.

Key words: cochlear implant; cross-modal plasticity; deaf white cat; deafness; deprivation; development

Significance Statement

In a common view, the "unused" auditory cortex of deaf individuals is reorganized to a compensatory sensory function during development. According to this view, cross-modal plasticity takes over the unused cortex and reassigns it to the remaining senses. Therefore, cross-modal plasticity might conflict with restoration of auditory function with cochlear implants. It is unclear whether the cross-modally reorganized auditory areas lose auditory responsiveness. We show that the presence of cross-modal plasticity in a higher-order auditory area does not reduce auditory responsiveness of that area. Visual reorganization was moderate, spatially scattered and there were no interactions between cross-modally reorganized visual and auditory inputs. These results indicate that cross-modal reorganization is less detrimental for neurosensory restoration than previously thought.

\section{Introduction}

Congenital sensory loss triggers cross-modal plasticity in the deprived sensory cortex (Rauschecker, 1995; Bavelier et al., 2006;

Received Jan. 5, 2016; revised March 22, 2016; accepted April 19, 2016.

Author contributions: R.L. and A.K. designed research; R.L., P.B., J.T., P.H., and A.K. performed research; S.G.L. contributed unpublished reagents/analytic tools; R.L. analyzed data; R.L., S.G.L., and A.K. wrote the paper.

This work was supported by the German Research Foundation (DFG, Cluster of Excellence Hearing4All). MED-EL $\mathrm{GmbH}$ (Innsbruck, Austria) provided the Cls for the study and supported Dr. J. Tillein. Christiane Sprenger designed modifications to the stereotactic frame and helped to design the visual stimulation set-up. We thank Daniela and Eddy Kühne for valuable technical support during the experiments.

The authors declare no competing financial interests.

This article is freely available online through the J Neurosci Author Open Choice option.
Stein and Stanford, 2008; Merabet and Pascual-Leone, 2010; Stein et al., 2014). Cross-modal reorganization is likely related to enhanced abilities in the remaining senses as best documented in deaf individuals (Neville and Lawson, 1987; Levänen et al., 1998;

Correspondence should be addressed to Rüdiger Land, Institute of AudioNeuroTechnology and Department of Experimental Otology, Clinics of Otolaryngology, Hannover Medical School, Stadtfelddamm 34, Hannover 30625, Germany. E-mail: land.ruediger@mh-hannover.de.

DOI:10.1523/JNEUROSCI.0046-16.2016 Copyright $\odot 2016$ Land et al.

This is an Open Access article distributed under the terms of the Creative Commons Attribution License Creative Commons Attribution 4.0 International, which permits unrestricted use, distribution and reproduction in any medium provided that the original work is properly attributed. 
Bavelier and Neville, 2002; Bavelier et al., 2006; Auer et al., 2007; Bottari et al., 2010, 2014).

A reorganization toward a new modality might interfere with restoration of the original sensory input and thus could close sensitive periods for the therapy, for example, with cochlear implants (CIs) (Kral, 2007). This is related to a fundamental question of developmental neuroscience: can experience cause a sensory area to change the major driving modality and thus restructure the brain's connectome extensively? A differential role of auditory areas in cross-modal reorganization has been demonstrated, with visual reorganization specific to higher-order auditory fields in congenital deafness (Kral et al., 2003; Lomber et al., 2010; Leonard et al., 2012). Although the primary auditory cortex has been the focus of many studies (for review, see Kral and Sharma, 2012), so far, no information is available on auditory responsiveness of higher-order auditory fields in congenital deafness. Furthermore, it remains unclear whether cross-modal responses are evenly distributed within a reorganized field or if reorganized neurons cluster spatially. To answer these questions, the present study determined the auditory responsiveness and the quantitative relation between visual responsiveness and original auditory responsiveness in a secondary sensory area known to take over a cross-modal behavioral function in deafness (Lomber et al., 2010), the auditory dorsal zone (DZ). The investigation was performed at the level of individual neurons using large-scale microelectrode mapping.

The functional impact of cross-modal reorganization on the restored auditory responsiveness using CIs on neuronal responses in area DZ was compared between adult hearing cats (HCs) and congenitally deaf cats (CDCs). To allow large-scale mapping of the whole field with multielectrode arrays, instead of searching the optimal stimulus (possible only in few neurons), we used stimuli likely to evoke responses in the majority of neurons related to the given modality and mapped the whole fields. We used an acute preparation, allowing us to separate reorganizations of bottom-up and lateral interactions from other influences. We recorded responses to both modalities at the same positions.

The present study demonstrates a modest visual cross-modal reorganization and a dominance of auditory responsiveness in the cross-modally reorganized DZ. The data do not indicate interference between the reorganized and original modality and show a substantial preservation of the original sensory inputs, even after cross-modal reorganization, with an absence of bimodal interactions.

\section{Materials and Methods}

Animals. Experiments were performed in five adult congenitally deaf white cats (Heid et al., 1998) and four adult hearing controls (>12 months of age, 7 female and 2 male). HCs had normal hearing with click-evoked auditory brainstem response (ABR) thresholds $<40 \mathrm{~dB}$ $\mathrm{SPL}_{\mathrm{pe}}$. CDCs had been identified from the colony of deaf white cats using a hearing screening with ABRs within the first 4 weeks after birth (Heid et al., 1998). HCs and CDCs lived in the same housing conditions. Experiments were approved by the local state authorities of Lower Saxony (LAVES, Oldenburg) and were performed in compliance with the guidelines of the European Community for the care and use of laboratory animals (EU VD 86/609/EEC) and the German Animal Welfare Act (TierSchG).

Surgical preparation and CIs. Animals were premedicated with $0.25 \mathrm{mg}$ of atropine intraperitoneally and then anesthetized with $24.5 \mathrm{mg} / \mathrm{kg}$ ketamine hydrochloride (Ketavet; Parker-Davis) and $1 \mathrm{mg} / \mathrm{kg}$ xylazine hydrochloride (Rompun 2\%; Bayer). The animals were then tracheotomized and artificially ventilated. During artificial ventilation, the anesthetic was switched to isoflurane (Lilly) and maintained throughout the surgical procedures at 1.3-1.5 volume percentage isoflurane concentration in a 1:2 mixture of $\mathrm{O}_{2} / \mathrm{N}_{2} \mathrm{O}$. Adequacy of anesthesia depth and the animals' physiological state was monitored by means of ECG, heart rate, end-tidal $\mathrm{CO}_{2}$, muscle tone, and EEG signals. End-tidal $\mathrm{CO}_{2}$ was maintained at $<4.5 \%$. Core temperature was kept $>37.5^{\circ} \mathrm{C}$ using a homeothermic blanket. Physiological state was additionally monitored by analyzing capillary blood every $12 \mathrm{~h}$ for blood gas concentration, $\mathrm{pH}$, bicarbonate concentration, base excess, glycemia, and oxygen saturation. A modified Ringer's solution containing bicarbonate (according to the base excess) was infused intravenously. Each animal's head was fixed in a stereotactic holder (Horsley-Clarke).

We then retested the hearing status in all animals by measuring ABR. For this purpose, a small trephination was drilled at the vertex and ABR responses were recorded with an epidural silver-ball electrode (diameter $\sim 1 \mathrm{~mm}$ ) referenced to a silver-wire neck electrode. HCs had click response thresholds $<40 \mathrm{~dB}$ SPL. The absence of ABR responses in deaf animals confirmed deafness as diagnosed during early hearing screening soon after birth (Fig. 1A).

Stimulation was performed using feline CIs (MED-EL, custom-made, five channels, distance between contacts $1 \mathrm{~mm}$; Fig. 1) inserted bilaterally through the round window into scalae tympani of both ears. This involved exposing both bullae and ear canals. To prevent electrophony in HCs, the hair cells were destroyed pharmacologically before cochlear implantation. This was achieved by intracochlear instillation of $300 \mu \mathrm{l}$ of $2.5 \%$ neomycin sulfate solution over a $5 \mathrm{~min}$ period and subsequent rinsing using Ringer's solution. The absence of hearing was subsequently confirmed by the absence of ABRs (Fig. 1). To test the functionality of the CIs and to determine the stimulation threshold, we then determined electrical ABRs (eABRs; Fig. 1). eABR thresholds were measured between the epidural silver electrode to a reference in the neck (amplification $100,000 \times$, sixth-order band-pass filter 10-10,000 Hz). Electrical brainstem responses were recorded for a biphasic pulse (200 $\mu \mathrm{s} /$ phase $)$ at different current levels with bipolar stimulation between all possible bipolar electrode contact combinations.

As in a previous study in a larger group of animals (Tillein et al., 2012), the eABR thresholds were not different between HCs and CDCs [Fig. 1D, HCs: left ear $154 \pm 43 \mu \mathrm{A}$; right ear: $139 \pm 28 \mu \mathrm{A}$; CDCs: left ear $161 \pm$ $79 \mu \mathrm{A}$; right ear $152 \pm 38 \mu \mathrm{A}$; Wilcoxon-Mann-Whitney (WMW) test, $\alpha=5 \%]$.

Recording of electrophysiological activity. For electrophysiological recording, a trephination above the lateral (suprasylvian) sulcus was performed, exposing the dorsal auditory cortex (DZ) on the lateral bank and visual medial suprasylvian sulcus areas (anterior and posterior medial suprasylvian sulcus areas, AMLS/PMLS, subsequently referred to jointly as MLS; Fig. 2A). The dura was opened and the cortex surface was covered with silicone oil. A modified Davies chamber was positioned around the trephination site to stabilize the cortex with a layer of agarose and a closure was created melted bone wax after the electrode arrays were set in place. Cortical activity was recorded with two linear 16 site multielectrode arrays for which the intersite distance was $150 \mu \mathrm{m}$, surface area 177 $\mu \mathrm{m}^{2}$, impedance 1-2 $\mathrm{M} \Omega$ (NeuroNexus). The multielectrode arrays were positioned and inserted using micromanipulators, which were attached to the stereotactic frame (TSE Systems). The penetration angle was kept constant throughout the experiment. At least one penetration in each investigated area was stained using DiI (1,1'-dioctadecyl-3,3,3', $3^{\prime}$ tetramethylindocarbocyanine perchlorate; Invitrogen) that was applied to the noncontact side of the multielectrode array using a micropipette (Eppendorf). An epidural vertex silver-ball electrode served as an electrical reference for both multi electrode arrays. The recorded signals were amplified 5000-10,000 times with a Neuralynx amplifier, band-pass filtered $(1 \mathrm{~Hz}-9 \mathrm{kHz})$, digitized (at a sampling rate of $30 \mathrm{kHz}$ ), and stored on a computer.

Mapping procedure and stimulation design. We mapped the dorsal auditory cortex and visual areas along the medial part of the suprasylvian sulcus in deaf and HCs (Fig. 1B). Multielectrode arrays were inserted on both sides of the sulcus at a distance of $\sim 500 \mu \mathrm{m}$ from the midline of the sulcus, thus penetrating the dorsal auditory cortex and the two visual area in MLS. With an intersite distance of $150 \mu \mathrm{m}$ and the uppermost site inserted just into the cortex, the tip of the electrode shank was inserted 
A

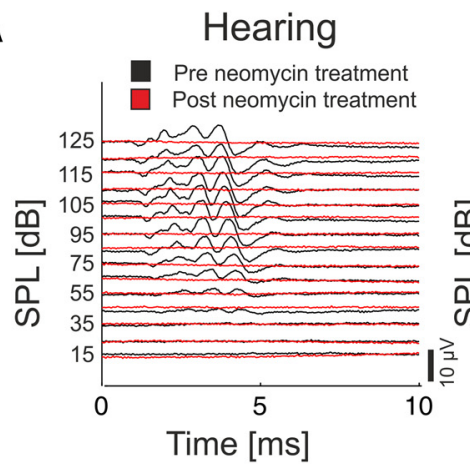

\section{B}

Deaf



C

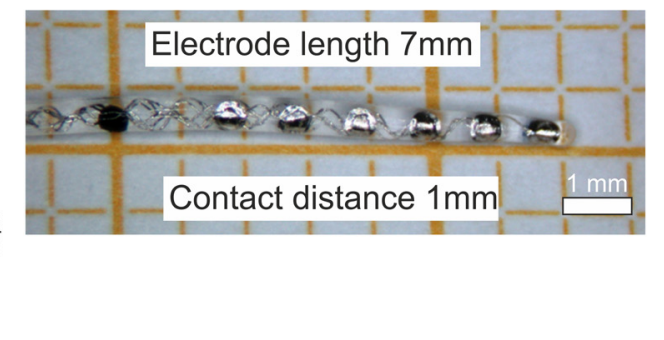

D

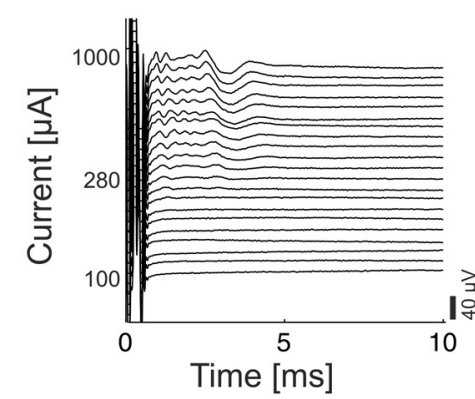

E

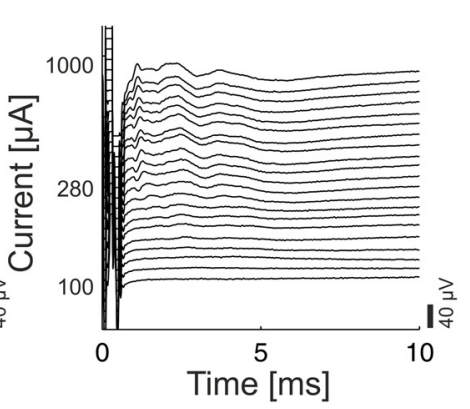

$\mathbf{F}$

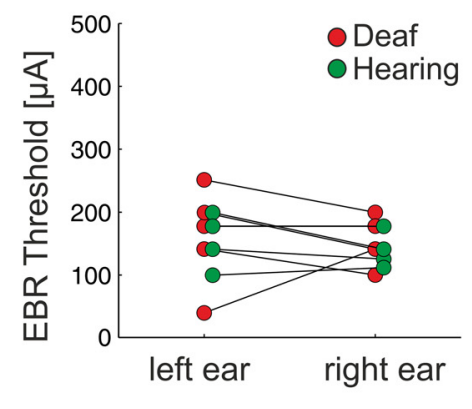

Figure 1. Cochlear implants in HCs and CDCS. $A$, In HCS, acoustic stimulation generated evoked brainstem responses that disappeared after destruction of hair cells by intracochlear application of neomycin. This treatment was required to eliminate electrophonic effects (see text for details). $\boldsymbol{B}, \ln C D C$, the conclusion of the juvenile hearing screening procedure was confirmed at the beginning of the acute experiments by an absence of brainstem responses for stimulation at high sound pressure levels. C, Cochlear implants used for the present experiments were donated by MED-EL. Stimulation was with apical-most and basal-most contact ( $5 \mathrm{~mm}$ distance) in wide bipolar configuration with charge-balanced biphasic pulses at $200 \mu \mathrm{s} / \mathrm{phase}$. D, eABRs in HCs after implantation. $\boldsymbol{E}$, eABRs in CDCs after implantation. $\boldsymbol{F}$, eABR thresholds (connected by the line) show no systematic difference in threshold between deaf and hearing animals or between the ears.

with a micromanipulator to a depth of $\sim 2400 \mu \mathrm{m}( \pm 100 \mu \mathrm{m})$ from the cortical surface. The depth or position of the multielectrode array was not changed after insertion to search for activity. At each penetration position and after the closure of the modified Davies chamber with agarose and bone wax, we allowed the multielectrode array to settle and stabilize the recordings for 10-20 min. Each block of sensory stimulation was initiated by $15 \mathrm{~min}$ of recording of spontaneous activity and was concluded by $15 \mathrm{~min}$ recording of spontaneous activity to exclude drifts of the general state of the animals. This approach allowed us to ensure a constant light anesthetic state. We paid attention to avoid deep anesthesia with burst suppression to prevent possible abnormal heteromodal responses in the cortex (Land et al., 2012). During the neuronal recordings, isoflurane concentration was reduced to $1.0-1.2$ volume percentage, and adequacy of anesthesia depth was monitored to ensure comparable anesthesia levels and to avoid periods of burst suppression.

The number of spontaneously active sites was similar for HCs and CDCs, with more spontaneous activity in the visual cortex than in the auditory dorsal cortex in both groups (CDCs: $63 \pm 7 \%$ in DZ vs $84 \pm 6 \%$ in MLS, WMW test, $p=0.03$; HCs: $53 \pm 5 \%$ in DZ vs $75 \pm 11 \%$ in MLS; WMW test, $p=0.029$ ).

We analyzed and included all electrode sites in the statistics. In the text, "position" refers to the penetration location in the cortex (Fig. 2B) and "site" refers to electrode sites deep in the cortex, of which there were 16 for each electrode array. In total, we analyzed 1440 recording sites (720 in the auditory and 720 in the visual cortex) in HCs and 1632 sites (816 in the visual and 816 in the auditory cortex) in CDCs.

Sensory stimulation. eABR thresholds were determined at the beginning of the experiment (Fig. 1). The eABR threshold of the respective ears then was used as a reference current level. Electrical stimulation was wide bipolar between the apical-most and the basal-most contact of the implant, covering cochlear positions with characteristic frequencies $>10$ $\mathrm{kHz}$ (Kral et al., 2009).

Auditory stimulation. For intracortical recordings, pulses were presented binaurally, from $2 \mathrm{~dB}$ below to $6 \mathrm{~dB}$ above the eABR threshold in
$1 \mathrm{~dB}$ steps for each ear. The electrical stimulus was a triplet of biphasic pulses (200 $\mu \mathrm{s} / \mathrm{phase}$ at 500 pulses/s, giving a total stimulation time of 4.4 $\mathrm{ms}$ ) applied in bipolar configuration between the basal-most electrode and the apical-most electrode of the CI. Pulse levels were randomized and the interstimulus intervals were $1000 \mathrm{~ms}$. Each electrical stimulus was repeated 30 times.

Visual stimulation. Visual stimuli were generated in MATLAB (The MathWorks) with the Psychophysics Toolbox (Brainard, 1997). Stimuli were presented on a TFT display (Model 2009wt; Dell) at a $28 \mathrm{~cm}$ distance in front of the contralateral eye. In analogy to the electrical pulse, we used a visual flash stimulus to study general visual responses. This stimulus is simple and broadly activates neurons in the visual system, both in the magnocellular and parvocellular subsystems. We presented $100 \mathrm{~ms}$ full-field flashes with positive contrast (white flash) or negative contrast (black flash). Each type of flash was repeated 50 times with an interstimulus interval of $1000 \mathrm{~ms}$ consisting of a gray background. Furthermore, to include apparent movement into the stimulus, square-wave phase reversal gratings of different orientations $\left(0^{\circ}, 45^{\circ}, 90^{\circ}, 135^{\circ}\right)$ and spatial frequencies $(0.1-2.0$ cycles/degree $)$ were used for visual stimulation.

Bimodal stimulation. To investigate interactions between the visual and auditory responses, bimodal stimulation was used. Visual stimulus (full-field flash, $16.7 \mathrm{~ms}$ duration, one frame, $60 \mathrm{~Hz}$ refresh rate) was combined with auditory stimulation (triplet of biphasic pulses, $200 \mu \mathrm{s} /$ phase, $500 \mathrm{~Hz}$ ) at $6 \mathrm{~dB}$ above threshold. The onset of the stimuli varied across a range from -30 to $30 \mathrm{~ms}$.

Histology. After the experiment, the animals were transcardially perfused. After thoracotomy, $0.5 \mathrm{ml}$ of heparin (Liquemin; Hoffman-La Roche) was injected into the left ventricle. Then, $2 \mathrm{~L}$ of phosphate buffer (0.1 M, pH 7.4) and $2 \mathrm{~L}$ of fixative (2.5\% glutaraldehyde and $2.0 \%$ formaldehyde) were infused transcardially with pressure $>100 \mathrm{mmHg}$. After $24 \mathrm{~h}$ of postfixation in $4 \%$ formaldehyde, the brain was excised from the skull, photographed, and a block containing the investigated cortical areas was cryoprotected in $30 \%$ sucrose, frozen, and cut in frontal plane 
A

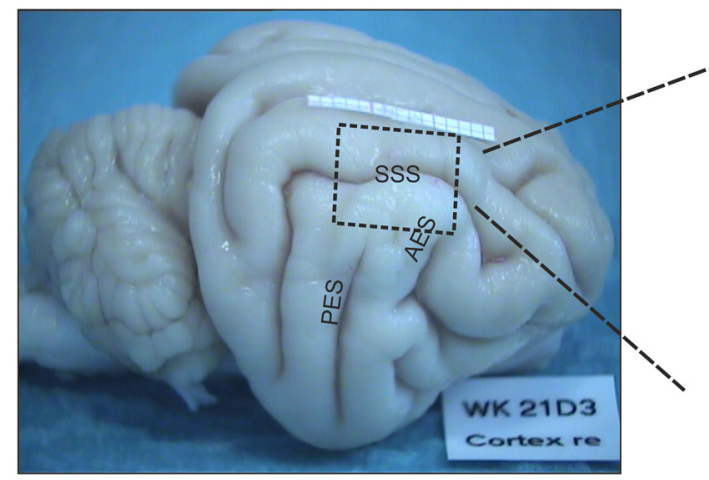

B

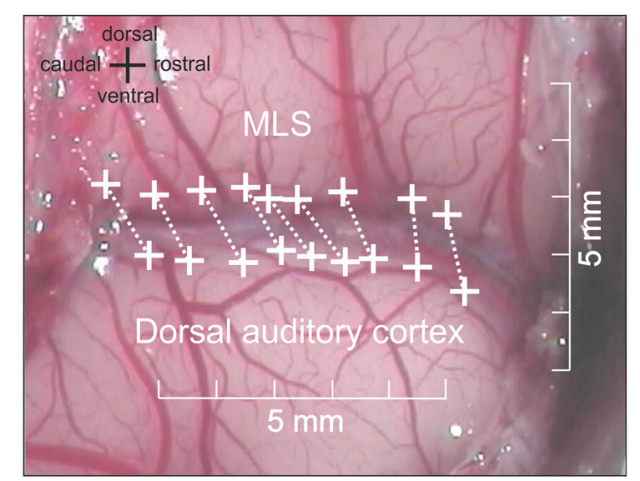

C

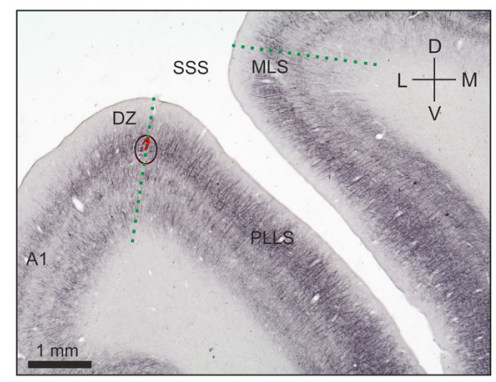

D


Figure 2. Mapping of auditory and visual areas in $C D C$ s and HCS. $A$, Lateral view of the brain of an adult CDC after the study. The dashed rectangle depicts the region of interest. Scale on the top of the brain: one division $\sim 1 \mathrm{~mm}$. $\boldsymbol{B}$, Surface of the depicted brain in $\boldsymbol{A}$ during the experiment. We studied the auditory DZ and lateral visual areas, which are located on either side of the suprasylvian sulcus. Sixteen-site multielectrode axial arrays were inserted along both sides within $1 \mathrm{~mm}$ of the middle suprasylvian sulcus in the rostrocaudal direction between the posterior ectosylvian sulcus (PES) and anterior ectosylvian sulcus (AES). Penetrations in the dorsal auditory cortex and the visual medial side of the suprasylvian sulcus are marked by white crosses; simultaneously recorded pairs are connected by a dashed white line. C, Coronal section of the investigated region after the experiment stained with SMI-32 to confirm histologically the position of penetrations within the intended cortical areas. Dotted lines (green) denote penetrations of the multielectrode arrays. In the DZ penetration, the red color within the circle is the overlay of the Dil deposit from the multielectrode array used to reconstruct the position and direction of the penetration photographed in the same section before SMI staining. $\boldsymbol{D}$, Overview of all positions of penetrations in the dorsal auditory cortex and visual MLS in adult CDCs $(n=5)$ and adult HCs $(n=4)$ arranged along a straightened suprasylvian sulcus (dashed black line) relative to the position of the PES. Different colors denote different cats. L, lateral; M, medial; D, dorsal; V, ventral; PLLS, posterior lateral suprasylvian sulcus area; SSS, suprasylvian sulcus.

in $50 \mu \mathrm{m}$ sections using a cryotome (Leica). The sections were first photographed in fluorescence mode to reveal the DiI (Keyence, BZ-9000). Subsequently, the sections were alternatively stained with Nissl and antibodies against SMI 32 (Mellott et al., 2010), allowing us to identify the borders of field A1, DZ, and lateral sulcus regions (LLS and MLS). All stained sections were then digitized and the penetrations were reconstructed (Keyence, BZ-9000). The DiI-stained penetrations were combined with photographs of SMI-32-stained (same) sections.

Data analysis. Multiunits (MUs) were derived by band-pass filtering the raw signal between $700 \mathrm{~Hz}$ and $9 \mathrm{kHz}$. First, we determined all spike activity with amplitudes that exceeded a fixed threshold of $50 \mu \mathrm{V}$ (amplifier noise level $<15 \mu \mathrm{V}$ ), separating large spikes. A fixed spike threshold was used to ensure comparability between groups and multiunit firing rates. We additionally analyzed "continuous multiunit activity" (cMUA), including the all spike amplitudes (also the so-called "hash") using the $700 \mathrm{~Hz}$ high-pass filtered, rectified, and squared signal without thresholding. This signal was denoted as cMUA.

Analysis of ongoing activity. To derive ongoing multiunit rate from 15 min intervals before and after stimulation, for each site, we randomly selected 100 intervals with a $1 \mathrm{~s}$ window length and calculated the mean rate of these 100 intervals for all sites. We subsequently excluded sites if firing rate was $<0.1 \mathrm{~Hz}$ during the entire period (nonactive sites).

Analysis of responses. Sites in CDCs and HCs were defined as responsive if neuronal activity was modulated by electrical stimulation via the CI satisfying a fixed statistical criterion for all sites (DZ and PMLS). Mean auditory responses were calculated for each of the 9 stimulation levels ( $-2 \mathrm{~dB}$ to $6 \mathrm{~dB}$ above the eABR threshold). Response strength was defined as the mean number of spikes in the interval $30 \mathrm{~ms}$ after stimulus onset; that is, the time window when auditory responses occurred. Auditory responses were ordered by stimulation level and the correlation coefficient between response strength and stimulation level was determined. If the coefficient was significant $(p<0.05)$ or if the unit significantly responded above baseline in five of the nine stimulation levels (two-sided $t$ test against baseline activity, $p<0.05$ ), then the site was considered responsive to auditory input. For all responsive sites, the response latency was defined as the peak of the response at $6 \mathrm{~dB}$ above threshold. Visual responsiveness was considered as present in those neurons that showed a significant increase in firing rate within the $60 \mathrm{~ms}$ after the stimulus ( $\alpha=5 \%$ ). Visually evoked activity was tested against baseline multiunit activity before stimulus onset. Both rates were compared with a two-sided $t$ test $(p<0.05)$ and, if found significant, were collected as a response for further analysis. Response latency was defined as peak latency.

Presence of bimodal enhancement was tested at those stimulus delays where peak responses overlapped. Quantification was performed using the enhancement index (EI) (Meredith and Stein, 1983)

$$
\text { E.I. }=\frac{V A-\max (V, A)}{\max (V, A)}
$$

where VA is the firing rate with bimodal stimulation, $V$ and $A$ are the firing rates of visual alone and auditory alone stimulation, respectively, and max denotes the maximum function. To determine the additive or superadditive character of bimodal responses, the additive index (AI) was also used (King and Palmer, 1985)

$$
\text { A.I. }=\frac{V A-(V+A)}{V+A}
$$

If not stated otherwise, all data are presented in the form of mean \pm SD. Data from animals were not pooled, statistical comparisons were per- 
A
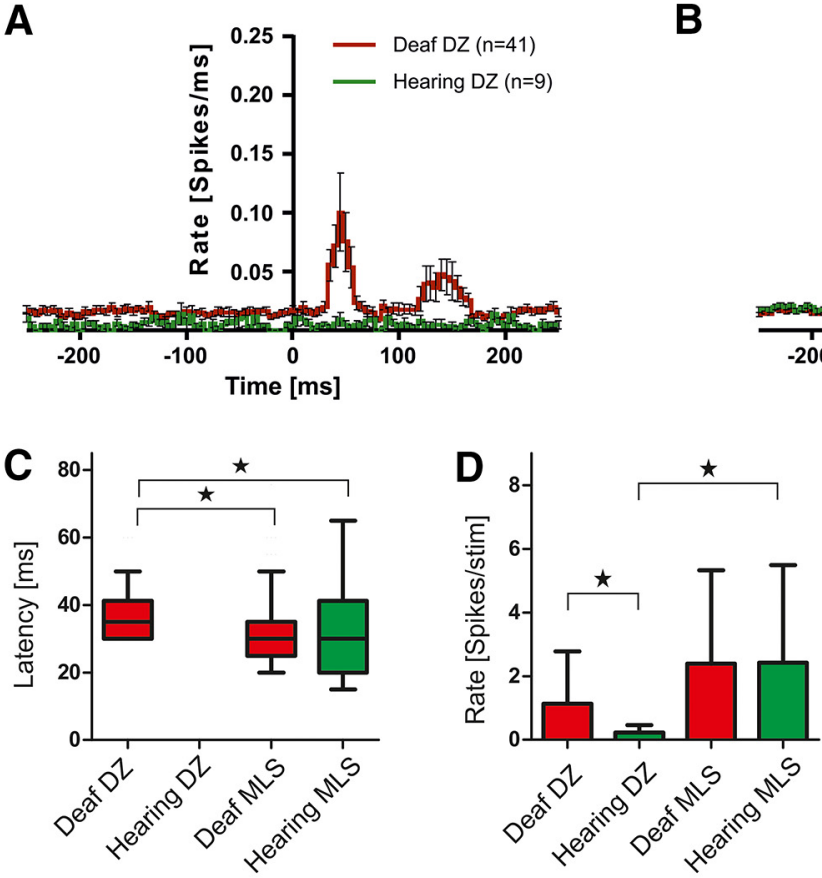

E
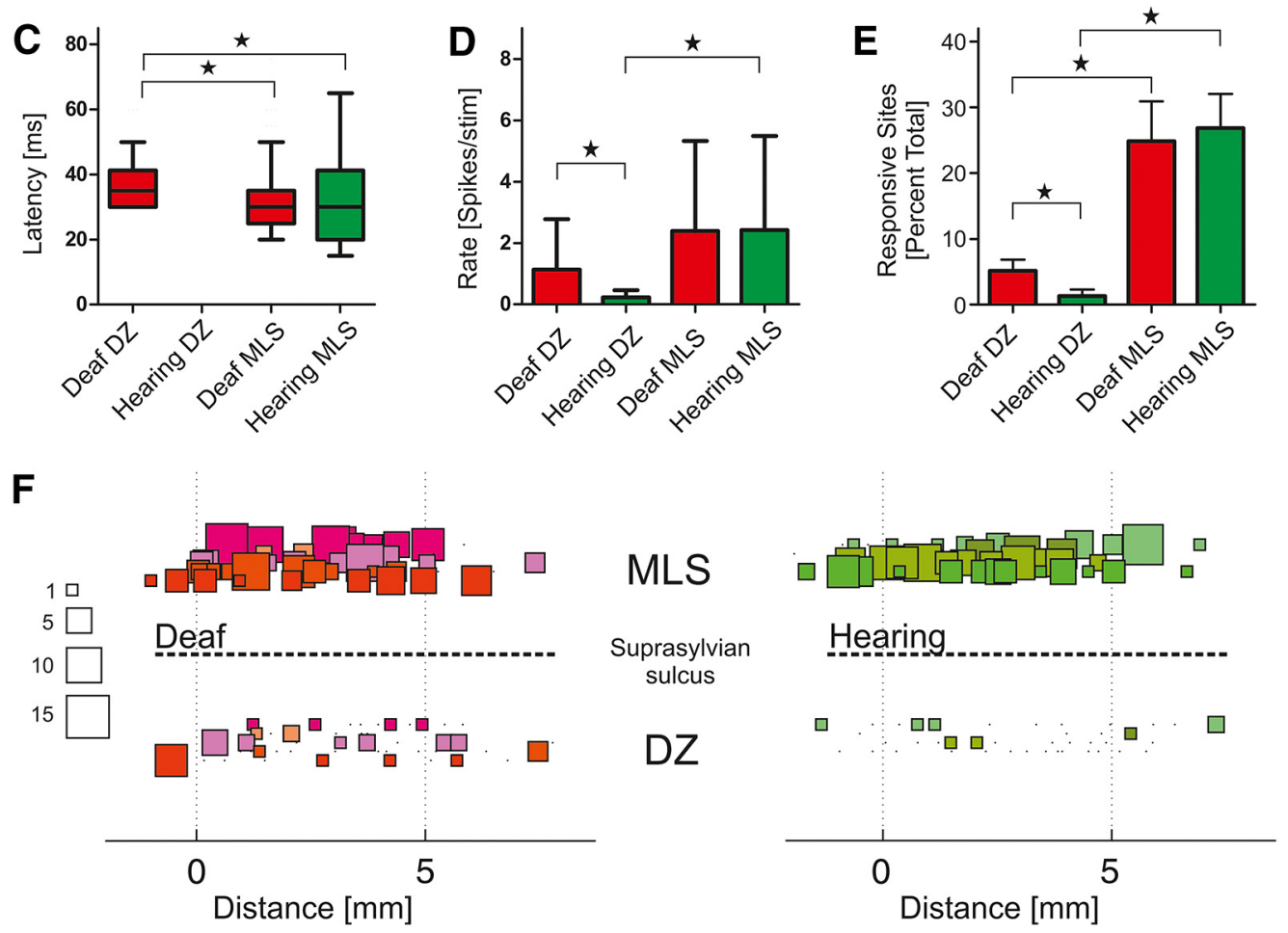

Figure 3. Visual responses in the dorsal auditory cortex of CDCS. $A$, Comparison of visual responses in the auditory DZ of CDCs and HCs. Shown are mean poststimulus time histograms with SEM error bars. Only deaf animals showed a visual response in DZ. B. Comparison of visual responses in the visual areas reveals no differences between congenital deaf and $H C s$. Onset and offset responses for the flashes are visible both in MLS and in DZ. Shown are mean poststimulus time histograms with SEM error bars. Similar visual responsiveness was observed in the visual areas of CDCS and HCs. C, Comparison of peak latencies of the visual responses in the dorsal auditory cortex and visual areas. Tukey boxplots show the median with 1.5 interquartile range whiskers. Deaf DZ responses had a slightly longer response latency. $\boldsymbol{D}$, Visually evoked firing rates in DZ were significantly higher in CDCs than in HCs. Firing rates were significantly higher in the MLS fields compared with DZ in HCS, but not in CDCS. Plots denote mean \pm SD error bars. $E$, Number of visually responsive sites was significantly higher in the dorsal auditory cortex of CDCs than in HCS. Plots denote mean with SD error bars. $F$, Responsiveness to flashes arranged along the straightened lateral sulcus (dashed line) in deaf and HCs (left vs right) relative to the dorsal end of the posterior ectosylvian sulcus (distance $=$ $0 \mathrm{~mm}$ ). Number of responsive sites per shank is depicted by the size of the rectangles and nonresponsive sites by small dots; animals are coded by color. Size-count examples are shown on the left side of the plot.

formed at the animal level (5 CDCs vs 4 HCs). We used a nonparametric two-tailed WMW test with 5\% significance level to compare data between cortical areas and between HCs and CDCs.

\section{Results}

The present study investigates the sensory function of a higher-order auditory area (DZ) in adult CDCs. Cooling inactivation of this area previously demonstrated a causal relation to supranormal visual behavior in CDCs (Lomber et al., 2010). Here, neuronal responses were collected simultaneously with two DiI-stained single-shank multielectrode arrays (16-channel) inserted to cover all cortical layers in DZ and the visual fields in the middle suprasylvian region (MLS; Palmer et al., 1978; Fig. 2). In total, we analyzed 1632 sites in CDCs $\left(n_{\text {visual }}=816\right.$ and $\left.n_{\text {auditory }}=816\right)$ and 1440 recording sites in HCs $\left(n_{\text {visual }}=720\right.$ and $\left.n_{\text {auditory }}=720\right)$.
Visual reorganization of the dorsal auditory cortex of CDCs

Visual stimuli allowing standardized large-scale mapping of cortical areas with multielectrode arrays (flashes and phasereversal gratings) were presented using a screen covering the frontal visual field.

Statistical significance of visual responsiveness was determined by comparing prestimulus and poststimulus firing rate. Visual responsiveness did not differ between flashes and gratings. Because flashes represented a stimulus better corresponding to the auditory stimulus, in the following, we focused on the responses to flash stimuli. From all responsive sites, mean time histograms were computed (Fig. $3 A, B$ ). Response to the visual flash showed prominent $\mathrm{ON}$ and OFF response typical for visual areas. A distinct difference between CDCs and HCs in visual responsiveness was only found in DZ (Fig. $3 A$ ), where CDCs 
exhibited visual responses, whereas HCs did not. In contrast, the visual areas showed similar visual responses in CDCs and $\mathrm{HCs}$ (Fig. 3B). This demonstrates a dissociation of the effect of congenital deafness on MLS neurons and DZ neurons.

The latencies of visual responses in the "deaf" DZ were higher than response latencies in the MLS, the control visual area (Fig. $3 C, \mathrm{CDC}_{\mathrm{DZ}={ }_{41}}$ : median $35 \mathrm{~ms}$ with median absolute deviation, $\mathrm{MAD}$, of $7.6 \mathrm{~ms}$; $\mathrm{CDCs}_{\mathrm{MLS}=165}$ : median $30 \mathrm{~ms}$ with MAD $7.3 \mathrm{~ms}$; $\mathrm{HC}_{\mathrm{MLS}=184}$ : median $30 \mathrm{~ms}$ with MAD $11.2 \mathrm{~ms}$; WMW test, $p=$ $0.005)$. The visual evoked firing rates in the deaf $\mathrm{DZ}$ were lower than the rates in the visual areas, but differed significantly from activity in the HCs, where no detectable evoked responses were present (Fig. $3 D, \mathrm{CDC}_{\mathrm{DZ}=41}: 1.1 \pm 1.6$ spikes/ stimulus; $\mathrm{HC}_{\mathrm{DZ}=9}: 0.2 \pm 0.2$ spikes/stimulus; WMW test, $p=$ 0.007). In contrast, in the visual cortex, latency and firing rate of responses did not differ significantly (Fig. 3C,D, $\mathrm{CDC}_{\mathrm{MLS}=165}: 2.4 \pm 2.9$ spikes/stimulus; $\mathrm{HC}_{\mathrm{MLS}=184}: 2.4 \pm 3.0$ spikes/stimulus, WMW test, $p=0.37$ ) and the latencies corresponded to those observed in MLS previously (Dinse and Krüger, 1994; Nowak and Bullier, 1997).

We found a moderate number of visually responsive recording sites in DZ of CDCs (Fig. 3E; CDC:5.1 \pm 3.0\%; HC:1.3 \pm $0.9 \%$; WMW test, $p=0.03$; for higher responsiveness with units with smaller amplitude, see below). Whereas visual responsiveness in DZ was thus emphasized in CDCs, visual responsiveness in the visual cortex was similar to that in HCs (Fig. 3E, CDC:24 \pm 13\%; HC:26 $\pm 10 \%$; WMW test, $p=0.85$ ). The overall number of DZ units responsive to visual stimulation in CDCs constituted $\sim 20 \%$ of visually responsive units in the visual areas. Together, these results demonstrate cross-modal reorganization in field DZ of CDCs, a possible correlate of previous behavioral observations (Lomber et al., 2010).

The position of visually responsive sites across the "deaf" auditory cortex did not show a systematic gradient (Fig. $3 F$ ). Visual responses in the deaf animals were sparsely distributed in the dorsal auditory cortex, whereas, in the visual cortex, visual responses were abundant both in CDCs and HCs (Fig. 3F).

In total, the absence of a difference in visual responsiveness in the control visual areas (MLS) between the investigated groups demonstrate that the general state of the animals was comparable between the two groups and cannot account for the difference in DZ responsiveness. Therefore, the difference in visual responsiveness in field DZ between HCs and CDCs was the result of the different hearing experience.

\section{Preserved auditory responsiveness in DZ}

To activate the auditory system and test auditory responsiveness, we used electrical stimulation with CIs in both groups of animals (Fig. 1). Implantation was performed acutely during the final experiments: none of the animals had previous experience with the electrical stimulation. However, HCs had normal hearing and their auditory system developed under the influence of hearing. Electrical "hearing thresholds" were quantified by eABRs (Fig. 1). For comparability, we also stimulated HCs with CIs (Fig. 1). Their hair cells were destroyed pharmacologically before implantation to prevent electrophonic responses.

The responsiveness to the auditory stimulus was again determined statistically and mean poststimulus time histograms were computed from responsive sites (Fig. 4A,B). CI-evoked responses were found in DZ of both CDCs and HCs (Fig. 4A,B). CDCs had slightly shorter auditory response latencies than $\mathrm{HCs}$ (Fig. $4 C, \mathrm{CDC}_{\mathrm{DZ}=149}$ : median $11 \mathrm{~ms}$ with MAD $3.7 \mathrm{~ms}$ and $\mathrm{HC}_{\mathrm{DZ}=113}$ median $12 \mathrm{~ms}$ with MAD $3.6 \mathrm{~ms}$; WMW test, $p=$
0.03 ), indicating a change in the response properties in the deaf DZ compared with HCs. CI-evoked firing rates in DZ of CDCs and $\mathrm{HCs}$ did not differ significantly (Fig. $4 D ; \mathrm{CDC}_{\mathrm{DZ}=149}: 0.69 \pm$ 0.7 spikes/stimulus; $\mathrm{HC}_{\mathrm{DZ}=113}: 0.64 \pm 0.65$ spikes/stimulus; WMW test, $p=0.74)$. We found no significant differences in the number of responsive sites in DZ between groups (Fig. $4 E$, CD$\mathrm{C}_{\mathrm{DZ}=149}: 20 \pm 9 \% ; \mathrm{HC}_{\mathrm{DZ}=113}: 16 \pm 7 \%$; WMW test, $p=0.38$ ). Auditory responsive sites were thus similarly present throughout the entire field DZ in both groups of animals (Fig. $4 F$ ); however, there was a tendency toward cluttering in the rostral part.

Both in HCs and CDCs, our selection criterion identified sites in the visual cortex as being auditory responsive. However, these were chance-level selections as a consequence of the automatic statistical criterion because poststimulus time histograms in the responsive sites did not reveal a response (Fig. $4 B, F$ ).

The results demonstrate that field DZ preserves its auditory responsiveness after congenital deafness despite cross-modal reorganization. The data show a dissociation of the effects of deafness on visual and auditory responsiveness in field DZ, with an increase in visual responsiveness in deaf DZ and no effect in MLS and similar auditory responsiveness in both investigated fields and both groups of animals, but moderate differences in the properties of the responses between deaf and hearing DZ (response latency).

Auditory responsiveness in DZ was similar in CDCs and HCs, with CDCs differing from HCs in the additional presence of visual responses. To exclude an influence of our spike selection criterion (large units), we additionally included an analysis of all units by analyzing the $700 \mathrm{~Hz}$ high-pass-filtered squared signal without thresholding (cMUA), including also small units.

With cMUA, the incidence of visual responses in DZ increased substantially, but the difference between the groups remained (Fig. 5A, CDC: $17 \pm 4 \%$, HC: $5 \pm 1 \%$; Mann-Whitney test, $p=$ $0.03)$. Also for cMUA, auditory responsive sites in the visually reorganized deaf DZ exceeded visually responsive neurons (Fig. $5 B$, cMUA: auditory: $48 \pm 23 \%$, visual: $17 \pm 9 \%$; MU: auditory: $20 \pm 9 \%$, visual: $5.1 \pm 4 \%$ ). Therefore, whereas the sensitivity of both measures differed, the auditory-responsive sites remained more frequent than visually responsive sites, so this observation was not due to the previous spike selection criterion (Fig. 5C).

\section{Absence of bimodal interactions}

Notably, only $1.4 \%(n=12)$ of all recorded sites in DZ of CDCs responded to both auditory and visual stimuli (i.e., were classified as bimodal units). We computed the bimodal EI for those stimulus timings where peak unimodal responses would overlap. The EI was between $-20 \%$ and $20 \%$ in 10 of 12 units with a mean of $-10 \pm 18(n=12)$. The bimodal AI was in most cases negative with a mean of $-20 \pm 15(n=12)$, indicating a subadditive character of the interactions. However, it should be kept in mind that these bimodal units represented only a negligible number of the total sites recorded and had rather weak responses.

In addition, we tested possible interactions of bimodal stimuli on firing rates within the visually reorganized deaf DZ in a more general approach. Because visual and auditory responses had different latencies and bimodal interactions depend on the timing between stimuli, visual and auditory stimuli were presented at different time delays relative to each other. In doing so, we measured bimodal interactions and responsiveness as a function of different time delays of bimodal stimuli. Provided a true modulation of one response by the other modality was present, a distinct peak in the responsiveness for certain time delays should 
A
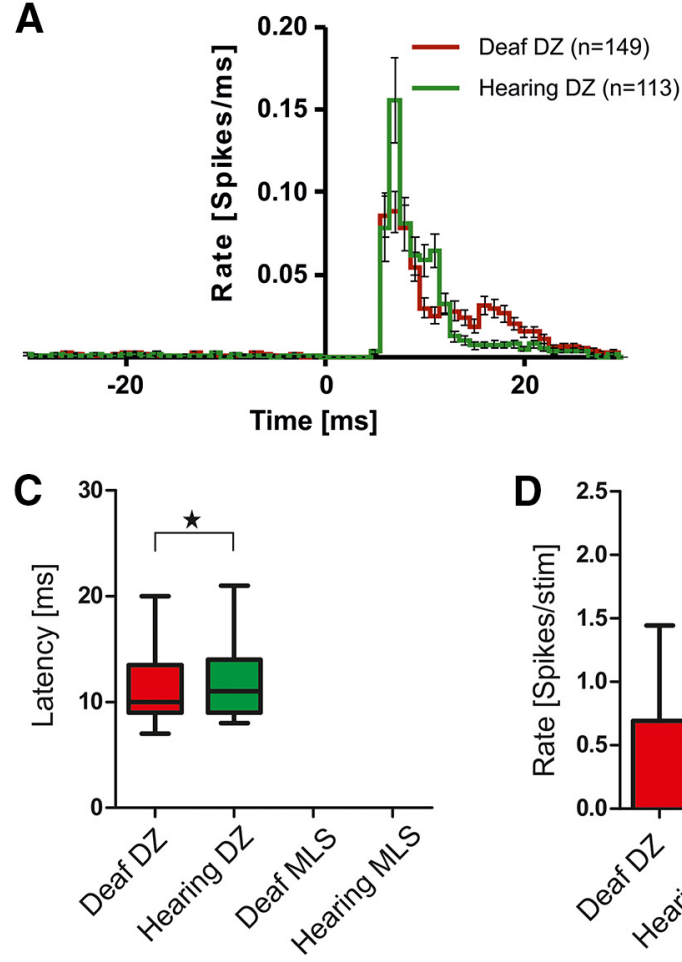

B

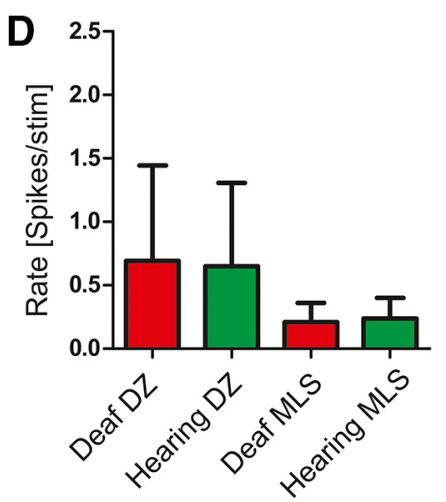

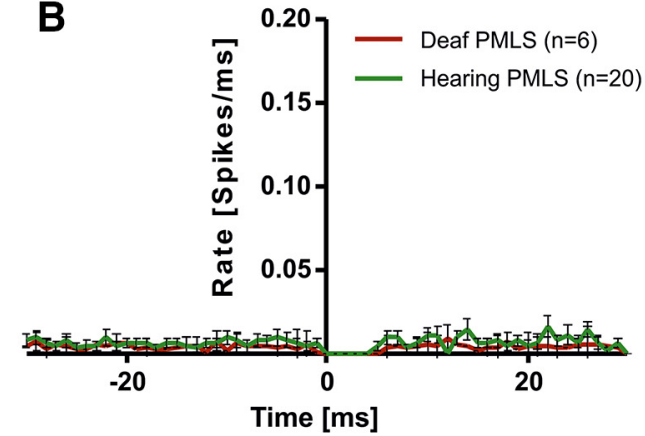



$\mathbf{F}$

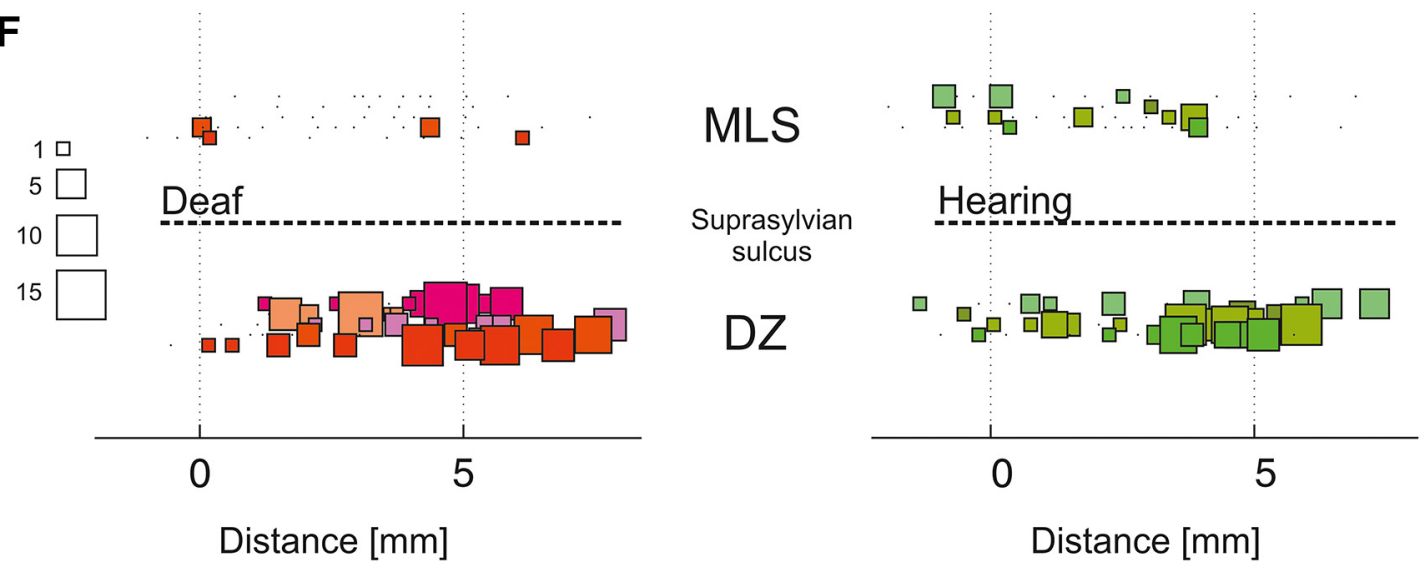

Figure 4. Cl eABRs in CDCs and HCS. A, Comparison of responses to Cl stimulation in DZ of CDCs and HCS. Poststimulus time histograms show mean responses with SEM error bars for responsive sites at $6 \mathrm{~dB}$ above eABR thresholds in the deaf animals. $B$, Visual areas in MLS did not respond to Cl stimulation. C, Comparison of latencies of responsive sites in CDCs and HCs. Tukey boxplots show the median with 1.5 interquartile range whiskers. $\boldsymbol{D}$, Response rates were similar in CDCs and HCs in all investigated fields. Plots denote mean with SD error bars. $\boldsymbol{E}$, Number of auditory-responsive sites was significantly higher in the auditory dorsal cortex than in the visual MLS region. Plots denote mean with SD error bars. $\boldsymbol{F}$, Responsiveness to flashes arranged along the straightened lateral sulcus (dashed line) in CDCs and HCs (left vs right) relative to the dorsal end of the posterior ectosylvian sulcus (distance $=0 \mathrm{~mm}$ ). Number of responsive sites is depicted by the size of the rectangles and nonresponsive sites by dots; animals are coded by color. Size-count examples are shown on the left side of the plot.

show up in this analysis. We inspected each site and then pooled the units to compare the mean results statistically.

First, we tested auditory neurons in the deaf DZ that did not respond to visual stimulation alone. These might respond to a combination of visual and auditory input. For the auditoryresponsive units, the auditory stimulus was kept constant at $6 \mathrm{~dB}$ above threshold and the visual flash (16.7 ms duration) was shifted in time relative to the auditory stimulus. The visual stimulus did not influence auditory responsiveness in the set of purely auditory-responsive units in the deaf DZ ( $n=100$ with response rate $>0.3$ spikes/stimulus). We computed the average for each cat and then tested the modulation by time delay (Fig. $5 D, n=5$ CDCs, comparing modulation of average responses for each cat, ANOVA, $\left.F_{(15,64)}=0.09, p=1\right)$. Subsequently, we compared nonresponding neurons $(n=670$ with response rate $<0.3$ spikes/ stimulus). Bimodal interactions might take place at subcortical levels and neurons that do not respond to a single condition may respond to a combination of stimuli. This was also not the case (Fig. $5 E, n=5 \mathrm{CDCs}$, comparing modulation of average responses of each cat, ANOVA, $\left.F_{(15,64)}=0.82, p=0.64\right)$. In HC, firing rates exhibited no relations to visual onset (data not shown). The last group comprised visually responsive neurons in the deaf DZ. Due to the presence of the electrical artifact from the CI stimulation, several stimulus time delays could not be tested because some units were lost due to blanking. In the remaining delays, a temporal shift of the auditory stimulus relative to the visual stimulus did not reveal any significant influence of the auditory stimulus on the visual response (Fig. $5 F ; n=5$ CDCs, comparing modulation of average responses of each cat, ANOVA, $\left.F_{(3,16)}=0.04, p=0.98\right)$. Therefore, the set of auditory- 
A

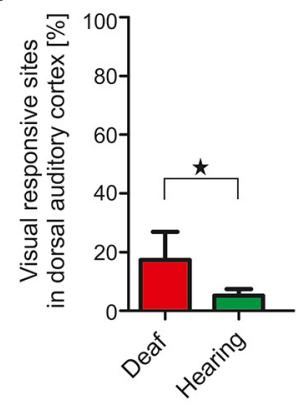

D



B

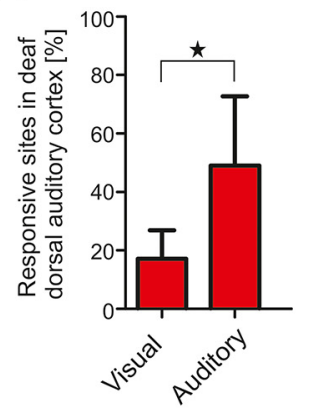

E

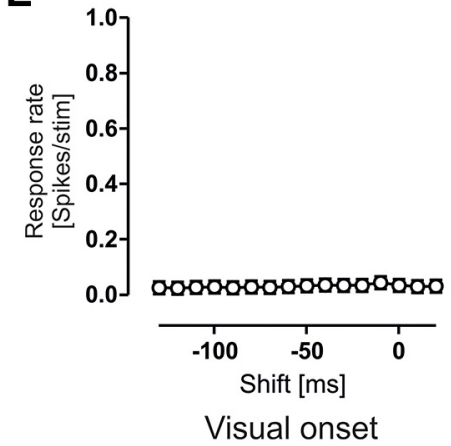

C

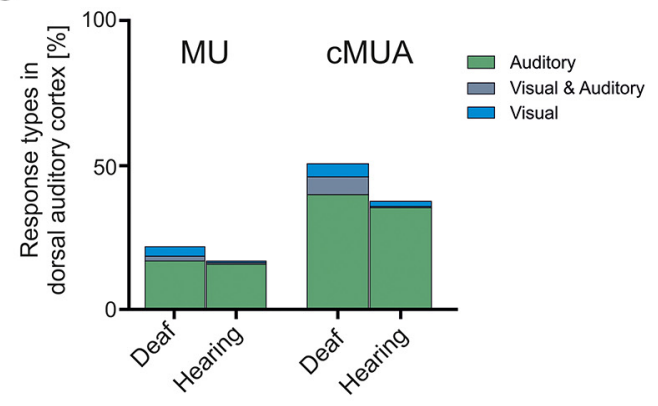

F



Figure 5. Absence of bimodal responses in the visually reorganized deaf DZ. A, cMUA responses (including all spikes) also reveal differences in visually responsive sites in the dorsal auditory cortex between CDCs and HCS. Plots denote mean with SD error bars. $\boldsymbol{B}$, Direct comparison of auditory and visual responses in the deaf auditory cortex. Responsiveness of the visually reorganized dorsal auditory cortex of $C D C$ s showed more auditory-responsive sites than visually responsive sites. Plots denote mean with SD error bars. $C$, Comparison of ratio between auditory responsive and visually responsive sites for MUA and CMUA. $\boldsymbol{D}$, Responsiveness to auditory stimulation was not affected by visual stimulation at different onset asynchronies. Plots denote mean with SD error bars. $\boldsymbol{E}$, Responsiveness of visual response was not affected by auditory stimulation at different onset asynchronies. Plots denote mean with SD error bars. $\boldsymbol{F}$, Nonresponsive sites were not affected by different stimulus asynchronies. Plots denote mean with SD error bars.

responsive sites and the set of visually responsive sites in the dorsal auditory cortex seem to be essentially distinct without interactions between sensory inputs when tested with electrical stimuli in the auditory domain.

\section{Discussion}

The present data demonstrate cross-modal reorganization in the secondary auditory field of CDCs, a possible correlate of previous behavioral observations. The cross-modal visual reorganization, however, did not reduce auditory responsiveness substantially even after long-term congenital deafness ( $>1$ year). Crossmodally responsive units were sparsely distributed in $\mathrm{DZ}$ and were approximately three times less frequent than auditory responsive units. The few units with audiovisual responses did not show bimodal enhancement.

The comprehensive mapping of auditory and visual fields demonstrated a dissociation of deprivation effects between the areas and sensory inputs: the higher-order auditory cortex developed visual responsiveness in CDCs. In contrast, there was no effect of lifelong auditory deprivation on the adjacent visual cortex. The data thus demonstrate a dissociation of the effect of deafness on responsiveness of auditory and visual areas.

Although cross-modal reorganization is well documented (Rauschecker, 1995; Bavelier et al., 2006; Auer et al., 2007; Merabet and Pascual-Leone, 2010; Ding et al., 2015) and related to enhanced abilities in the remaining senses in deaf individuals (Neville and Lawson, 1987; Levänen et al., 1998; Bavelier et al., 2006; Bottari et al., 2014; Stein et al., 2014), the original function of the reorganized neural tissue has not been explored before at the level of neurons. The absence of adverse effects of cross- modal reorganization on auditory responsiveness has implications for visual communication before cochlear implantation in children born deaf.

\section{Methodological considerations}

The cross-modally reorganized DZ is known to be involved in visual motion detection (Lomber et al., 2010). Here, we used flashes and phase-reversal gratings known to evoke strong responses in motion-sensitive and pattern-sensitive neurons (Kral et al., 2003). Strong responsiveness with such stimuli in neighboring motion-sensitive visual areas confirmed their effectiveness in driving the neurons (cf. Dinse and Krüger, 1994; Nowak and Bullier, 1997). The responsiveness to grating and flashes was similar. Although we cannot exclude that a more specific visual stimulus could have caused stronger visual responsiveness in field DZ, this argument can never be disproven. The present standardized approach is optimal for large-scale mapping purposes. Furthermore, the flash stimulus is a good visual correlate of the wide bipolar electrical stimulus used for auditory stimulation, allowing direct comparison of the intermodal balance in responsiveness.

We did not investigate the detailed functional properties of CI responses. The present study, as a first step, focused on responsiveness; more detailed future studies might reveal further differences in auditory properties of deaf DZ beyond the latency differences observed here. Here, the deaf brains were untrained, completely naive to auditory stimuli, and still stronger auditory than visual responsiveness was observed in their field DZ. Hearing experience with CI would likely further increase the auditory 
responsiveness in DZ, as demonstrated in field A1 (Klinke et al., 1999; Kral et al., 2006, 2013).

Bimodal supraadditive interactions follow the law of inverse effectiveness (Stein and Stanford, 2008). With respect to bimodal interactions, the small dynamic range with electrical stimulation is thus a limiting factor. However, because the present study even did not observe additive interactions, this small dynamic range cannot explain the absence of bimodal interactions.

\section{Cross-modal reorganization in congenital deafness}

Cross-modal reorganization in DZ corresponds to data from humans (Finney et al., 2001, 2003; Bavelier et al., 2006). Ectopic subcortical and lateral projections into DZ (from the adjacent MLS, area 7, and areas 19/20/21) are present in CDCs (Barone et al., 2013). Cross-modal visual responses in the deaf DZ had latencies slightly longer than responses in the adjacent MLS (Palmer et al., 1978), consistent with a direct connection from MLS to the deaf DZ in CDCs. Visually responsive sites in the deaf DZ were moderate in number and accounted for $\sim 30 \%$ of the auditory-responsive sites. The present findings are thus consistent with the perceptual-narrowing hypothesis (Ghazanfar and Schroeder, 2006; Lewkowicz, 2014; Maurer and Werker, 2014) because such sparse responsiveness may result from patchy exuberant connections that appear developmentally and are normally pruned during postnatal life (Innocenti and Price, 2005; Innocenti, 2011).

Here, we have provided data for visual flash stimulation because this stimulus is independent of the receptive field and other properties of the individual neuron. With regard to the moderate $5 \%$ or $17 \%$ of visually responsive sites in the crossmodally reorganized cortex (compared with $25 \%$ or $60 \%$ of auditory-responsive sites), it needs to be emphasized that it is not clear what minimal amount of cross-modal reorganization is necessary to enhance performance. Distributed attentional and top-down modulations are likely to complement (boost) the cross-modal responsiveness described here. Conflicting findings in the direction of change of sensory capabilities in blind or deaf individuals, which can range from an increase to a decrease (Pavani and Bottari, 2012), indicate that cortical cross-modal reorganization may be variable depending on a given individual's (top-down) compensation strategy.

\section{Auditory activation of the secondary auditory cortex in deafness}

The deaf DZ remained responsive to the auditory input. The auditory responsiveness was not different from HC, demonstrating that the secondary auditory cortex develops its basic functional responsiveness even in absence of any hearing. This corresponds to persistence of basic functionality within the deprived sense of congenitally sensory-deprived humans (Collignon et al., 2009; Leonard et al., 2012; Striem-Amit et al., 2012a, 2012b; Cardin et al., 2013, 2016; Gandhi et al., 2015). The present study provides the physiological substrate of this phenomenon. Supporting this, sighted and congenitally blind subjects show specificity for motion or object form in the occipital cortex regardless of input modality, even in absence of visual experience (Voss and Zatorre, 2012; Peelen et al., 2014).

Some studies have reported negative interference from visual inputs for auditory processing in deaf subjects (Doucet et al., 2006; Buckley and Tobey, 2011; Sandmann et al., 2012; Chen et al., 2016). Conversely, beneficial cross-modal effects for speech understanding have been reported, mainly in the postlingually deaf (Giraud et al., 2000; Rouger et al., 2007; Stropahl et al.,
2015). These latter studies, however, investigated postlingually deafened groups, whereas the present study investigated the congenitally deaf.

The increase in visual responsiveness in the present study was not related quantitatively to a similar decrease in auditory responsiveness. Therefore, units that were visually responsive were not strongly coupled to the auditory system. We did not find bimodal interactions in the deaf DZ. In cats, visual deprivation interferes with development of multimodal integration (Wallace et al., 2004, 2006; Carriere et al., 2007, 2008). The absence of vision during development interferes with the development of multimodal interactions in the superior colliculus, but less with visual responsiveness (Yu et al., 2013; Stein et al., 2014). Even rearing under omnidirectional noise has a similar effect (Xu et al., 2014). Furthermore, early visual deprivation has detrimental effects on multimodal integration in association area 7 (adjoining the MLS areas; Carlson et al., 1987). A loss of bimodal interactions and a dominance of visual inputs have been documented in prelingually deaf children after cochlear implantation (Schorr et al., 2005). Moreover, congenital cataracts prevented audiovisual integration in humans (Guerreiro et al., 2015). The absence of bimodal interactions in the present study is explicable by the absence of early auditory experience that is essential for development of audiovisual interactions.

Visual recruitment of higher-order auditory areas might close the sensitive periods for therapy of deafness (Kral, 2007; Lomber et al., 2010). Even though some neurons took over visual functions and were auditory nonresponsive in the present study, the general auditory responsiveness was not affected significantly. Therefore, the major limiting factors after sensitive periods appear to be the developmentally reduced plasticity and central processing deficits within the auditory modality itself (Kral et al., 2002, 2009, 2013; Fallon et al., 2009). It needs to be emphasized that absence of a difference in auditory DZ responsiveness in the present study does not demonstrate the absence of functional auditory deficits. Such deficits have been described numerous times in the auditory system (for review, see Kral, 2013) and are likely also present in the dorsal auditory cortex. The present study, however, did focus only on responsiveness and its balance between visual and auditory stimulation.

Bimodal training has improved auditory spatial localization and changed auditory responses in field A1 of deaf ferrets equipped with CIs (Isaiah et al., 2014). This demonstrates that after focused training bimodal processing can become beneficial for some (but maybe not all, see Schorr et al., 2005; Guerreiro et al., 2015) functions after restoration of hearing, probably via topdown influences from the frontal cortex (Isaiah et al., 2014). The present data are consistent with these findings.

The present study has an important translational aspect. Communication before cochlear implantation in deaf children is an important predictive factor of implantation outcomes (Tait et al., 2000). Some argue that deaf children should communicate via enhanced focus on visual input (cued speech or sign language) before cochlear implantation (Lyness et al., 2013; Campbell et al., 2014; Mellon et al., 2015). However, many clinicians are concerned with potential negative interactions between modalities (Geers et al., 2011; Mellon et al., 2015). Indeed, the cross-modally reorganized units in the present study did not respond to auditory stimulation. However, they represented only a minor portion of neurons in DZ. Consequently, the present data disprove that cross-modal reorganization eliminates functional auditory inputs to the higher-order dorsal auditory cortex. 


\section{References}

Auer ET Jr, Bernstein LE, Sungkarat W, Singh M (2007) Vibrotactile activation of the auditory cortices in deaf versus hearing adults. Neuroreport 18:645-648. CrossRef Medline

Barone P, Lacassagne L, Kral A (2013) Reorganization of the connectivity of cortical field DZ in congenitally deaf cat. PLoS One 8:e60093. CrossRef Medline

Bavelier D, Neville HJ (2002) Cross-modal plasticity: where and how? Nat Rev Neurosci 3:443-452. Medline

Bavelier D, Dye MW, Hauser PC (2006) Do deaf individuals see better? Trends Cogn Sci 10:512-518. CrossRef Medline

Bottari D, Nava E, Ley P, Pavani F (2010) Enhanced reactivity to visual stimuli in deaf individuals. Restor Neurol Neurosci 28:167-179. CrossRef Medline

Bottari D, Heimler B, Caclin A, Dalmolin A, Giard MH, Pavani F (2014) Visual change detection recruits auditory cortices in early deafness. Neuroimage 94:172-184. CrossRef Medline

Brainard DH (1997) The Psychophysics Toolbox. Spat Vis 10:433-436. CrossRef Medline

Buckley KA, Tobey EA (2011) Cross-modal plasticity and speech perception in pre- and postlingually deaf cochlear implant users. Ear Hear 32:2-15. CrossRef Medline

Campbell R, MacSweeney M, Woll B (2014) Cochlear implantation (CI) for prelingual deafness: the relevance of studies of brain organization and the role of first language acquisition in considering outcome success. Front Hum Neurosci 8:834. CrossRef Medline

Cardin V, Orfanidou E, Rönnberg J, Capek CM, Rudner M, Woll B (2013) Dissociating cognitive and sensory neural plasticity in human superior temporal cortex. Nat Commun 4:1473. CrossRef Medline

Cardin V, Smittenaar RC, Orfanidou E, Rönnberg J, Capek CM, Rudner M, Woll B (2016) Differential activity in Heschl's gyrus between deaf and hearing individuals is due to auditory deprivation rather than language modality. Neuroimage 124:96-106. CrossRef Medline

Carlson S, Pertovaara A, Tanila H (1987) Late effects of early binocular visual deprivation on the function of Brodmann's area 7 of monkeys (Macaca arctoides). Brain Res 430:101-111. Medline

Carriere BN, Royal DW, Perrault TJ, Morrison SP, Vaughan JW, Stein BE, Wallace MT (2007) Visual deprivation alters the development of cortical multisensory integration. J Neurophysiol 98:2858-2867. CrossRef Medline

Carriere BN, Royal DW, Wallace MT (2008) Spatial heterogeneity of cortical receptive fields and its impact on multisensory interactions. J Neurophysiol 99:2357-2368. CrossRef Medline

Chen LC, Sandmann P, Thorne JD, Bleichner MG, Debener S (2016) Crossmodal functional reorganization of visual and auditory cortex in adult cochlear implant users identified with fNIRS. Neural Plast 2016:4382656. CrossRef Medline

Collignon O, Voss P, Lassonde M, Lepore F (2009) Cross-modal plasticity for the spatial processing of sounds in visually deprived subjects. Exp Brain Res 192:343-358. CrossRef Medline

Ding H, Qin W, Liang M, Ming D, Wan B, Li Q, Yu C (2015) Cross-modal activation of auditory regions during visuo-spatial working memory in early deafness. Brain 138:2750-2765. CrossRef Medline

Dinse HR, Krüger K (1994) The timing of processing along the visual pathway in the cat. Neuroreport 5:893-897. CrossRef Medline

Doucet ME, Bergeron F, Lassonde M, Ferron P, Lepore F (2006) Crossmodal reorganization and speech perception in cochlear implant users. Brain 129:3376-3383. CrossRef Medline

Fallon JB, Irvine DR, Shepherd RK (2009) Cochlear implant use following neonatal deafness influences the cochleotopic organization of the primary auditory cortex in cats. J Comp Neurol 512:101-114. CrossRef Medline

Finney EM, Fine I, Dobkins KR (2001) Visual stimuli activate auditory cortex in the deaf. Nat Neurosci 4:1171-1173. CrossRef Medline

Finney EM, Clementz BA, Hickok G, Dobkins KR (2003) Visual stimuli activate auditory cortex in deaf subjects: evidence from MEG. Neuroreport 14:1425-1427. CrossRef Medline

Gandhi T, Kalia A, Ganesh S, Sinha P (2015) Immediate susceptibility to visual illusions after sight onset. Curr Biol 25:R358-R359. CrossRef Medline

Geers AE, Strube MJ, Tobey EA, Pisoni DB, Moog JS (2011) Epilogue: factors contributing to long-term outcomes of cochlear implantation in early childhood. Ear Hear 32:84S-92S. CrossRef Medline
Ghazanfar AA, Schroeder CE (2006) Is neocortex essentially multisensory? Trends Cogn Sci 10:278-285. CrossRef Medline

Giraud AL, Truy E, Frackowiak RS, Grégoire MC, Pujol JF, Collet L (2000) Differential recruitment of the speech processing system in healthy subjects and rehabilitated cochlear implant patients. Brain 123:1391-1402. CrossRef Medline

Guerreiro MJ, Putzar L, Röder B (2015) The effect of early visual deprivation on the neural bases of multisensory processing. Brain 138: 1499-1504. CrossRef Medline

Heid S, Hartmann R, Klinke R (1998) A model for prelingual deafness, the congenitally deaf white cat-population statistics and degenerative changes. Hear Res 115:101-112. CrossRef Medline

Innocenti GM (2011) Development and evolution: two determinants of cortical connectivity. Prog Brain Res 189:65-75. CrossRef Medline

Innocenti GM, Price DJ (2005) Exuberance in the development of cortical networks. Nat Rev Neurosci 6:955-965. CrossRef Medline

Isaiah A, Vongpaisal T, King AJ, Hartley DE (2014) Multisensory training improves auditory spatial processing following bilateral cochlear implantation. J Neurosci 34:11119-11130. CrossRef Medline

King AJ, Palmer AR (1985) Integration of visual and auditory information in bimodal neurones in the guinea-pig superior colliculus. Exp Brain Res 60:492-500. Medline

Klinke R, Kral A, Heid S, Tillein J, Hartmann R (1999) Recruitment of the auditory cortex in congenitally deaf cats by long- term cochlear electrostimulation. Science 285:1729-1733. CrossRef Medline

Kral A (2007) Unimodal and crossmodal plasticity in the "deaf" auditory cortex. Int J Audiol 46:479-493. CrossRef Medline

Kral A (2013) Auditory critical periods: a review from system's perspective. Neuroscience 247:117-133. CrossRef Medline

Kral A, Sharma A (2012) Developmental neuroplasticity after cochlear implantation. Trends Neurosci 35:111-122. CrossRef Medline

Kral A, Hartmann R, Tillein J, Heid S, Klinke R (2002) Hearing after congenital deafness: central auditory plasticity and sensory deprivation. Cereb Cortex 12:797-807. CrossRef Medline

Kral A, Schröder JH, Klinke R, Engel AK (2003) Absence of cross-modal reorganization in the primary auditory cortex of congenitally deaf cats. Exp Brain Res 153:605-613. CrossRef Medline

Kral A, Tillein J, Heid S, Klinke R, Hartmann R (2006) Cochlear implants: cortical plasticity in congenital deprivation. Prog Brain Res 157:283-313. CrossRef Medline

Kral A, Tillein J, Hubka P, Schiemann D, Heid S, Hartmann R, Engel AK (2009) Spatiotemporal patterns of cortical activity with bilateral cochlear implants in congenital deafness. J Neurosci 29:811-827. CrossRef Medline

Kral A, Hubka P, Heid S, Tillein J (2013) Single-sided deafness leads to unilateral aural preference within an early sensitive period. Brain 136: 180-193. CrossRef Medline

Land R, Engler G, Kral A, Engel AK (2012) Auditory evoked bursts in mouse visual cortex during isoflurane anesthesia. PLoS One 7:e49855. CrossRef Medline

Leonard MK, Ferjan Ramirez N, Torres C, Travis KE, Hatrak M, Mayberry RI, Halgren E (2012) Signed words in the congenitally deaf evoke typical late lexicosemantic responses with no early visual responses in left superior temporal cortex. J Neurosci 32:9700-9705. CrossRef Medline

Levänen S, Jousmäki V, Hari R (1998) Vibration-induced auditory-cortex activation in a congenitally deaf adult. Curr Biol 8:869-872. CrossRef Medline

Lewkowicz DJ (2014) Early experience and multisensory perceptual narrowing. Dev Psychobiol 56:292-315. CrossRef Medline

Lomber SG, Meredith MA, Kral A (2010) Cross-modal plasticity in specific auditory cortices underlies visual compensations in the deaf. Nat Neurosci 13:1421-1427. CrossRef Medline

Lyness CR, Woll B, Campbell R, Cardin V (2013) How does visual language affect crossmodal plasticity and cochlear implant success? Neurosci Biobehav Rev 37:2621-2630. CrossRef Medline

Maurer D, Werker JF (2014) Perceptual narrowing during infancy: a comparison of language and faces. Dev Psychobiol 56:154-178. CrossRef Medline

Mellon NK, Niparko JK, Rathmann C, Mathur G, Humphries T, Napoli DJ, Handley T, Scambler S, Lantos JD (2015) Should all deaf children learn sign language? Pediatrics 136:170-176. CrossRef Medline

Mellott JG, Van der Gucht E, Lee CC, Carrasco A, Winer JA, Lomber SG 
(2010) Areas of cat auditory cortex as defined by neurofilament proteins expressing SMI-32. Hear Res 267:119-136. CrossRef Medline

Merabet LB, Pascual-Leone A (2010) Neural reorganization following sensory loss: the opportunity of change. Nat Rev Neurosci 11:44-52. CrossRef Medline

Meredith MA, Stein BE (1983) Interactions among converging sensory inputs in the superior colliculus. Science 221:389-391. CrossRef Medline

Neville HJ, Lawson D (1987) Attention to central and peripheral visual space in a movement detection task: an event-related potential and behavioral study. II. Congenitally deaf adults. Brain Res 405:268-283. CrossRef Medline

Nowak LG, Bullier J (1997) The timing of information transfer in the visual system. In: Cerebral cortex (Rockland KS, Kaas JH, Peters A, eds), pp 205-241. New York: Plenum.

Palmer LA, Rosenquist AC, Tusa RJ (1978) The retinotopic organization of lateral suprasylvian visual areas in the cat. J Comp Neurol 177:237-256. CrossRef Medline

Pavani F, Bottari D (2012) Visual abilities in individuals with profound deafness a critical review. In: The neural bases of multisensory processes (Murray MM, Wallace MT, eds). Boca Raton, FL: CRC.

Peelen MV, He C, Han Z, Caramazza A, Bi Y (2014) Nonvisual and visual object shape representations in occipitotemporal cortex: evidence from congenitally blind and sighted adults. J Neurosci 34:163-170. CrossRef Medline

Rauschecker JP (1995) Compensatory plasticity and sensory substitution in the cerebral cortex. Trends Neurosci 18:36-43. CrossRef Medline

Rouger J, Lagleyre S, Fraysse B, Deneve S, Deguine O, Barone P (2007) Evidence that cochlear-implanted deaf patients are better multisensory integrators. Proc Natl Acad Sci U S A 104:7295-7300. CrossRef Medline

Sandmann P, Dillier N, Eichele T, Meyer M, Kegel A, Pascual-Marqui RD, Marcar VL, Jäncke L, Debener S (2012) Visual activation of auditory cortex reflects maladaptive plasticity in cochlear implant users. Brain 135: 555-568. CrossRef Medline

Schorr EA, Fox NA, van Wassenhove V, Knudsen EI (2005) Auditory-visual fusion in speech perception in children with cochlear implants. Proc Natl Acad Sci U S A 102:18748-18750. CrossRef Medline
Stein BE, Stanford TR (2008) Multisensory integration: current issues from the perspective of the single neuron. Nat Rev Neurosci 9:255-266. CrossRef Medline

Stein BE, Stanford TR, Rowland BA (2014) Development of multisensory integration from the perspective of the individual neuron. Nat Rev Neurosci 15:520-535. CrossRef Medline

Striem-Amit E, Cohen L, Dehaene S, Amedi A (2012a) Reading with sounds: sensory substitution selectively activates the visual word form area in the blind. Neuron 76:640-652. CrossRef Medline

Striem-Amit E, Dakwar O, Reich L, Amedi A (2012b) The large-scale organization of "visual" streams emerges without visual experience. Cereb Cortex 22:1698-1709. CrossRef Medline

Stropahl M, Plotz K, Schönfeld R, Lenarz T, Sandmann P, Yovel G, De Vos M, Debener S (2015) Cross-modal reorganization in cochlear implant users: Auditory cortex contributes to visual face processing. Neuroimage 121:159-170. CrossRef Medline

Tait M, Lutman ME, Robinson K (2000) Preimplant measures of preverbal communicative behavior as predictors of cochlear implant outcomes in children. Ear Hear 21:18-24. CrossRef Medline

Tillein J, Heid S, Lang E, Hartmann R, Kral A (2012) Development of brainstem-evoked responses in congenital auditory deprivation. Neural Plast 2012:182767. CrossRef Medline

Voss P, Zatorre RJ (2012) Organization and reorganization of sensorydeprived cortex. Curr Biol 22:R168-R173. CrossRef Medline

Wallace MT, Perrault TJ Jr, Hairston WD, Stein BE (2004) Visual experience is necessary for the development of multisensory integration. J Neurosci 24:9580-9584. CrossRef Medline

Wallace MT, Carriere BN, Perrault TJ Jr, Vaughan JW, Stein BE (2006) The development of cortical multisensory integration. J Neurosci 26: 11844-11849. CrossRef Medline

Xu J, Yu L, Rowland BA, Stanford TR, Stein BE (2014) Noise-rearing disrupts the maturation of multisensory integration. Eur J Neurosci 39: 602-613. CrossRef Medline

Yu L, Xu J, Rowland BA, Stein BE (2013) Development of cortical influences on superior colliculus multisensory neurons: effects of dark-rearing. Eur J Neurosci 37:1594-1601. CrossRef Medline 